\title{
Double core hole spectroscopy with synchrotron radiation
}

F. Penent ${ }^{1,2^{*}}$, M. Nakano ${ }^{3,4}$, M. Tashiro ${ }^{5}$, T. P. Grozdanov ${ }^{6}$, M. Žitnik ${ }^{7}$, K. Bučar ${ }^{7}$, S. Carniato ${ }^{1,2}$, P. Selles ${ }^{1,2}$, L. Andric ${ }^{1,2}$, P. Lablanquie ${ }^{1,2}$, J. Palaudoux ${ }^{1,2}$, E. Shigemasa ${ }^{5}$, H. Iwayama ${ }^{5}$, Y. Hikosaka $^{8}$, K. Soejima ${ }^{8}$, I.H. Suzuki ${ }^{3}$, N. Berrah ${ }^{9}$, A. H. Wuosmaa ${ }^{9}$, T. Kaneyasu ${ }^{10}$ and K. Ito ${ }^{3}$

${ }^{1}$ Sorbonne Université, UPMC, Université Paris 06, LCPMR, 11 rue P. et M. Curie, 75231 Paris Cedex 05, France.

${ }^{2}$ CNRS, LCPMR (UMR 7614), 11 rue P. et M. Curie, 75231 Paris Cedex 05, France.

${ }^{3}$ Photon Factory, Institute of Materials Structure Science, Oho, Tsukuba 305-0801, Japan.

${ }^{4}$ Department of Chemistry, Tokyo Institute of Technology, O-okayama, Tokyo 152-8551, Japan

${ }^{5}$ Institute for Molecular Science, Okazaki 444-8585, Japan.

${ }^{6}$ Institute of Physics, University of Belgrade, Pregrevica118, 11080 Belgrade, Serbia.

${ }^{7}$ Jožef Stefan Institute, P. O. Box 3000, SI-1001 Ljubljana, Slovenia.

${ }^{8}$ Department of Environmental Science, Niigata University, Niigata 950-2181, Japan

${ }^{9}$ University of Connecticut, Physics Department, , Storrs, CT 06269- USA

${ }^{10}$ SAGA Light Source, 8-7 Yayoigaoka, Tosu, Saga 841-0005, Japan

*Corresponding author, email: francis.penent@upmc.fr

Summary: In the last five years, a strong experimental and theoretical activity has been dedicated to the study of inner-shell double photoionization. Almost simultaneously, between 2009 and 2011, different experiments were performed using two different light sources: well established third generation synchrotron light sources that exist for more than two decades and recently developed $\mathrm{x}$-ray free electron lasers $(\mathrm{X}-\mathrm{FEL})$ that provide brightness about 10 orders of magnitude higher. The high photon flux of X-FEL has made possible the successive absorption of many $x$-ray photons by inner-shell electrons in femtosecond timescale. The possibility to create multiple inner-shell vacancies with this new X-FEL light source has resuscitated "prophetic" 25 years old calculations by Cederbaum et al. [1] [2] concerning double K-shell ionization that could be of great interest when conventional Electron Spectroscopy for Chemical Analysis (ESCA) finds some limitations. As a feedback, this has stimulated new theoretical developments. On the other hand, it was considered practically out of reach to observe such states with conventional third generation synchrotron light sources due to the very weak probability to eject two inner-shell electrons by a single photon when the dominant process, that could mask everything else, is single inner-shell ionization. However, due to the development of very efficient detection techniques, it was also possible to perform spectroscopy of double corehole states with excellent accuracy using third generation synchrotron sources.

In this paper we give an outlook, on the basis of recently published results, of the complementary of these light sources for double core-hole spectroscopy. Some new developments and results are also presented with a prospective of what could be done in the future with the technical breakthrough that will be necessary to improve further our understanding of chemical analysis.

Introduction:

Since 2009, a great interest has been devoted to the study of double core-hole ionization of atoms and molecules in the gas phase using X-FEL and synchrotron light sources. Such studies 
have a strong interest for understanding inner-shell electron correlation in atomic and molecular species and could have some practical applications for chemical analysis.

Electron spectroscopy for Chemical analysis (ESCA) [3] is a powerful and sensitive tool to characterize atomic species and their chemical environment. However, in 1986, Cederbaum et al [1] [2] anticipated that double core-hole ionization could lead to new possibilities for the characterization of molecules. Taking the example of pure hydrocarbon molecules $\left(\mathrm{C}_{2} \mathrm{H}_{2 n}(n=1\right.$ 3), $\left.\mathrm{C}_{6} \mathrm{H}_{6} \ldots\right)$, where the chemical environment is too similar to induce a strong shift in the K-shell ionization energy, it was shown that double core hole ionization energy would be very sensitive to the bond-length when different atoms are core-ionized simultaneously. At this time, the experimental demonstration of such predictions was out of reach and it was latter, with the arrival of a new light source: the x-ray Free Electron Laser (X-FEL), that the experiments appeared feasible due to the possibility of successive absorption of two (or even more) photons by inner-shell electrons. We will give a brief summary of these experiments in the following paragraph.

At the same time, our group [4] and Feifel's group [5] were developing experiments using magnetic bottle electron spectrometers on synchrotron centers that were both initiated by Eland's original set-up for the study of multiple ionization [6]. The improvement of electron detection efficiencies ( $4 \pi$ collection angle and high efficiency ( $>70 \%$ ) micro-channel plate (MCP) detectors) and coincidence techniques (up to 5 electrons in coincidence [7], reduced electronic dead-time to a few ns...) together with the enhanced performances of third generation synchrotron sources and beamlines, allowed the observation of single photon double core-hole ionization [4]. We further developed these studies and obtained new results that complement the results obtained on X-FEL. We will give a summary of our experimental achievements in this field [4] [8] [9] [10] and present some new results for a few other molecules we have studied.

Multiple and double core-hole ionization with X-FEL sources:

In atomic and molecular physics, multiphoton processes require high photon fluence and were, for a long time, restricted to visible and infrared laser studies. They have opened new research fields as above threshold ionization (ATI) [11], High Harmonics Generation (HHG) [12], attophysics [13], to mention only a few...

The recent apparition of X-FELs based on linear accelerators producing electrons bunches of about $10 \mathrm{GeV}$ and long undulators able to generate Self-Amplification of Spontaneous Emission (SASE) [14] [15], has, somehow, extended the possibility of multiphoton processes to the VUV and x-ray domains with peak powers of about $10 \mathrm{GW}$ and pulse duration of a few to hundreds of fs. However, while simultaneous absorption of a few IR or visible photons is necessary to ionize an atom, a single x-ray photon can ionize an inner-shell electron and, when a few photons are absorbed sequentially, this leads to multiple inner-shell ionization. The probability of simultaneous two-photon (or multiphoton) processes, well established with visible and IR lasers, remains however small. Only recently [16], evidence of two-photon simultaneous absorption at FERMI was performed to excite helium $1 \mathrm{D}^{\mathrm{e}}$ doubly excited states with two $30 \mathrm{eV}$ photons as it was possible with visible lasers for two-photon Doppler-free spectroscopy [17] [18].Simultaneous two-photon absorption is also involved in the ionization from $\mathrm{Ne}^{8+}$ to $\mathrm{Ne}^{9+}$ at LCLS [19] after sequential 1s ionization and Auger processes in $\mathrm{Ne}$.

With X-FEL, the photon density at the focal point is of the order of magnitude of the density of a solid. For solid targets, the x-ray range allows deep penetration and induces a strong heating to create dense plasmas in fs timescale that open new perspectives [20].

For the gas phase experiments we are interested in, such high photon density can (multiply) ionize all the atoms (molecules) present in the photon beam. Consequently, many electrons and ions are produced for every laser shot. This has led to previously inaccessible experimental studies. Since the dominant ionization in the soft $x$-ray domain 
is K-shell ionization (for second row element), the succession of ionization processes followed by Auger decay can lead to complete stripping of atoms and molecules as it was observed in $\mathrm{Ne}$ [21] and $\mathrm{N}_{2}$ [22] when the X-FEL pulse duration is longer ( 280fs) than the Auger decay time. Conversely, when the X-FEL pulse duration becomes shorter than the Auger lifetime, typically $5 \mathrm{fs}$ as it is possible at LCLS, only the "initial" K-shell electrons are ejected as in the $\mathrm{CO}[23]$ and $\mathrm{N}_{2}$ [24] cases because the K-shell cannot be refilled fast enough by Auger decay.

Such very high ionization efficiencies give rise to many possible processes (different charge states of the ions are produced) that can make the experiment sometimes difficult to interpret and call for technical breakthrough in data collection and analysis. A common tool on synchrotron centers is electron spectroscopy with hemispherical high performance analyzers. A serious difficulty with X-FEL may result from the space charge of ions in the interaction volume that could shift the electron energy by a few eV (the ponderomotive shift of the electrons remains small for short wavelengths even at such photon densities). To avoid this, the target gas density must be kept low enough (in the $10^{-5} \mathrm{~Pa}$ pressure range in the interaction region implying ultrahigh vacuum conditions for the base vacuum) to ionize only tens of atoms or molecules per pulse to limit the space charge effects. Consequently, due to the moderate repetition rate (from 10 to $100 \mathrm{~Hz}$ ) of the present XFEL sources a relatively long acquisition time (considering the availability of $X-F E L$ for users) and sophisticated data treatment are necessary [25] for each laser shot. These technical constraints did not prevent however to obtain impressive results for a lot of molecules [26].

The possibility of multiple inner-shell ionization and particularly of double K-shell ionization has been demonstrated [22] [23] and has recalled in the forefront the 1986's Cerderbaum et al's predictions concerning double core-hole spectroscopy [1] [2]. To interpret and predict the experimental spectra, new calculations [27] [28] became necessary stimulating further activity is this field.

For the molecules of interest containing $\mathrm{C}, \mathrm{N}$ and $\mathrm{O}$ atoms [26], the dominant decay channel after K-shell ionization is Auger decay. On the other hand, for a long time, the main diagnostic of double core hole states (mostly in heavier atoms: $Z>12$ ) that could be produced by different processes [29] [30] was X-ray spectroscopy with the observation of hypersatellite $x$-ray lines [31] [32]. The combination of $X$-FEL to produce double corehole states in dense plasmas with $X$-ray spectroscopy of such states should have a strong interest for plasma diagnostic since the corresponding hypersatellite radiation will not be reabsorbed as the $K_{\alpha}$ line would be.

However as is was mentioned in Cederbaum's article [1], the most sensitive process to the chemical bond length is not the observation of double K-shell ionization on the same atom (also called single site double core hole: $\mathrm{ss}-\mathrm{DCH}$ or shortly $\mathrm{K}^{-2}$ ) but the twosite double core-holes (ts- $\mathrm{DCH}$, shortly $\mathrm{K}^{-1} \mathrm{~K}^{-1}$ ). Although the sequential absorption of two photons by two K-shell electrons can occur on any atom in a molecule, the specific signature of the ts-DCH states is more difficult to obtain. While the hypersatellite Auger lines provide a good identification of ss-DCH states because the energy of the corresponding Auger electron is much higher than the "normal" Auger lines following single K-shell ionization, this is no longer the case for Auger decay of ts-DCH states because Auger lines fall in the same energy range than normal Auger lines. Hence, the experimental observation of ts-DCH states in the electron spectra is more difficult and is based on the signal difference between the photoelectron and Auger spectra obtained with the X-FEL focused and de-focused to subtract single core ionization spectra and reveal the quadratic (and higher) processes corresponding to the absorption of two (or more) photons. A first demonstration was done for the $\mathrm{CO}$ molecule [23] and latter extended to a series of molecules [26]. A good theoretical support is necessary to disentangle the complete spectra that show additional peaks due to absorption of a second photon after Auger decay of the core-shell created by the first absorbed photon 
(photoionization-Auger-Photoionization (PAP) process... and beyond). (see Fig 6\&7 in ref. [25]).

To go beyond the measurement of single photoelectron or Auger spectra, the covariance mapping technique [33] has also been adapted to extract electron correlation maps when electron coincidences techniques are not possible due to the present low FEL repetition rate. This method has been applied successfully to the Neon atom [34] with some adaptations - partial covariance mapping [35] - due to the inherent fluctuations of the pulse intensity and wavelength in SASE mode of the X-FEL. However, such technique cannot correlate more than 3 electrons [36] while, to identify properly DCH processes that lead to the emission of 2 photoelectrons and 2 Auger electrons, it would be necessary to correlate 4 electrons.

At the moment, it seems difficult to claim that X-ray two-photon photoelectron spectroscopy (XTPPS) [28] has become a routine technique on X-FEL light sources and a breakthrough in experimental techniques will be necessary to go well beyond the present work [19-22]. The straightforward way to disentangle all the different processes relies on coincident detection of all the electrons created by the multiple ionization of a single molecule. Coincidence techniques are perfectly suited for synchrotron light because the photon density is much lower and ionization events can be easily kept at a level where ionization of two atoms by the same photon bunch is negligible. The high repetition rate of the synchrotron (about $1 \mathrm{MHz}$ in single bunch mode) allows keeping the count rates to a few tens of $\mathrm{KHz}$ with negligible random coincidences. This will be detailed latter on. Adaptation of coincidence techniques to X-FEL would require the presence of no more than one atom (molecule) at the focus to avoid random coincidences. This needs ultra-high vacuum conditions with the target gas in and/or taking only the signal from a very limited region in the focus. Although such conditions can be achieved [37] and allow ion/ion coincidences, the limiting factor will be the repetition rate to get enough statistics in a "reasonable" time.

Double core-hole spectroscopy using synchrotron radiation

Synchrotron light sources deliver much lower photon peak intensities than X-FEL and double core-hole ionization cannot result from the absorption of two photons from the same light pulse (which have typically 20-50ps duration) consequently the only way is single photon double core ionization. This is possible due to electron correlation in an atom or a molecule but the cross-section for this process is generally weak. Consequently, a very efficient detection system is necessary and the magnetic bottle spectrometer fulfills the necessary conditions. In the last 12 years, it has proved to be very efficient to study valence double photoionization of many molecules [38] but also: double Auger decay in rare gases [39] [40] and molecules [41], triple ionization [42] [43], $4 \mathrm{~d}$ double-core ionization in Xe [44], thanks to the possibility to detect 2, 3 or 4 electrons in coincidence.

Following these studies, double $\mathrm{K}$-shell ionization in molecules with $\mathrm{C}, \mathrm{N}$ and $\mathrm{O}$ atoms was finally observed by our group [8] [4] and by Feifel's group [5] although it was previously claimed that :" the probability to produce a two-site double core hole state with one photon absorption is practically zero at third-generation synchrotrons due to low $x$ ray intensities" [27]. "Practically zero" means in fact a $\sim 10^{-3}$ fraction of single K-shell ionization for $\mathrm{K}^{-2}$ process [4] and $\sim 10^{-5}$ for $\mathrm{K}^{-1} \mathrm{~K}^{-1}$ [9] and the very high efficiency of the magnetic bottle allows such observations. These results were followed by many others on different molecules [9] [45] [46].

In single photon double K-shell ionization processes two photoelectrons are ejected in a first step and followed by sequential Auger emission of two electrons. The most direct way to characterize such processes consists in detecting those four electron in coincidence to obtain both the spectroscopy of $\mathrm{DCH}$ states and their specific Auger decay [8]. Less restricting conditions (2 photoelectrons +1 hypersatellite Auger electron) 
can allow also good characterization of these states [5]. The coincidence detection of all the electrons gives a lot of information that are not easily accessible with X-FEL experiments. The energy sharing between the two photoelectrons can be determined and the state specific cascade Auger decay is also observed. The stability of the photon energy and the good energy resolution of $\sim 2 \mathrm{~m}$ long magnetic bottle allow also high resolution spectroscopy of $\mathrm{DCH}$ states.

In the following we will describe the experiments we have done on synchrotron centers that allowed us to perform double core hole spectroscopy $\left(\mathrm{K}^{-2}, \mathrm{~K}^{-1} \mathrm{~K}^{-1}\right)$ of a few simple molecules $\left(\mathrm{N}_{2}, \mathrm{CO}, \mathrm{CO}_{2}, \mathrm{O}_{2}, \mathrm{H}_{2} \mathrm{O}, \mathrm{C}_{n} \mathrm{H}_{2 n}(\mathrm{n}=1-3) \ldots\right)$. A brief summary of our previous publications [4] [8] [9] will be given and additional results on new molecules: $\mathrm{C}_{3} \mathrm{H}_{6}$ (propylene), and ethyl trifluoroacetate ("Siegbahn ESCA" molecule), obtained with improved performances of the experimental set-up, will be detailed. In addition to double $\mathrm{K}$-shell ionization, a special attention will be given to simultaneous $\mathrm{K}$-shell ionization/Kshell excitation (in short $\mathrm{K}^{-2} \mathrm{~V}$ ) process that provides also a strong interest [10] [47] [48].

We give first a complete description of our experimental set-up.

\section{Experiment:}

The experiments were performed at the undulator beamlines BL-16A of the Photon Factory (PF) between 2009 and 2011, and TEMPO (2010) and SEXTANTS [49] beamlines (since 2011) at SOLEIL. Those beamlines deliver photons up to $1.5 \mathrm{keV}$ that are necessary to double ionize two K-shell electrons in $\mathrm{C}, \mathrm{N}$ and $\mathrm{O}$ atoms. The PF and SOLEIL synchrotrons were operated in single-bunch mode (respective revolution period of $624 \mathrm{~ns}$ and $1184 \mathrm{~ns}$ ) to allow electron time-of-flight measurements. Top-up refilling of the rings (stored ion current of $50 \mathrm{~mA}$ at PF and $15 \mathrm{~mA}$ at SOLEIL) ensured long term stability of the experimental conditions. The set-up at PF and SOLEIL are equivalent, they consist in long (2.5 or $2 \mathrm{~m}$ ) magnetic bottles electron time-of-flight spectrometers with a strong cylindrical-conical $(\varnothing 25 \mathrm{~mm}-\mathrm{L} 40 \mathrm{~mm})$ shaped permanent magnet (NdBFe or SmCo) that gives a magnetic field of about $0.5 \mathrm{~T}$ at $1 \mathrm{~mm}$ from the tip of the cone, that decreases quickly ( 100Gauss at $2 \mathrm{~cm})$. The strong B-field gradient creates a magnetic mirror that collects electrons on a full $4 \pi$ solid angle and parallelizes the electron trajectories in a few $\mathrm{cm}$. A solenoid wounded on the flight tube creates a B-field of about 10 Gauss that guides the electrons towards the detector. The detector is a high efficiency (>70\%) [50] micro-channelplates (MCP) assembly (Z-stack) that allows the detection of successive electrons with a dead time down to 6 ns (due to TDC) that will be further reduced to $3 \mathrm{~ns}$. The $50 \Omega$ impedance matching is critical to avoid signal rebounds that could mimic a second electron at about 10 to 15 ns (depending of cables lengths). Unfortunately, such rebound cannot be completely avoided and appear as a 5 to $10 \%$ fraction of the main pulse. Due to pulse height distribution, a compromise is necessary to reject such rebounds by setting the discriminator threshold high enough without losing significant detection efficiency. In our first measurements [4] this was not always possible and the option was to broaden the NIM $(-0.8 \mathrm{~V})$ electron signal after the discriminator to $\sim 15 \mathrm{~ns}$ to suppress rebounds. The dead time became hence $15 \mathrm{~ns}$. The time-of-flight of each electron with respect to the incident light pulse (provided by the ring clock) is converted by a multi-hit time to digital converter with a resolution of $120 \mathrm{ps}$ [51]. The conversion process is triggered by the detection of a first electron. The electron signal is sent in a channel of the TDC and the ring clock in another one. The data accumulation (in list mode) and the analysis have been detailed elsewhere [52]. Owing to the process of interest, the data are filtered with respect to the number of electrons: 2 for single Kshell ionization, 3 or 4 for DCH spectroscopy. Since the KLL Auger electrons are fast enough (from $\sim 200$ to $\sim 500 \mathrm{eV}$ for $\mathrm{C}, \mathrm{N}$ and O) their time-of-flight (TOF) is shorter ( 150$230 \mathrm{~ns})$ than the single bunch period of about $1 \mu \mathrm{s}$. Hence, it was not necessary to use a chopper [52] to extend the light period to $12 \mu \mathrm{s}$ in order to have absolute time-of-flight determination even for the slowest electrons (TOF up to $\sim 5 \mu$ s with a repelling potential of $-0.5 \mathrm{~V}$ on the magnet). The detection of the fast Auger electron selects unambiguously 
the ionizing light pulse and allows the determination of the absolute time of flight of the slower electrons resulting from the same multiple ionization event.

Electron time-of-flight to energy conversion was achieved by measuring photoelectrons of known kinetic energy ( $\mathrm{He}$ or $\operatorname{Ar}(2 \mathrm{p})$ photoelectrons) -after precise calibration of the monochromator on well-known atomic or molecular absorption resonances $\left(\mathrm{Ar}, \mathrm{O}_{2} \ldots\right)$ or absorption edges ( $\mathrm{Co}_{3}, \mathrm{Cu}_{3}$ and $\mathrm{Gd} \mathrm{M}_{5}$ ) using appropriate thin filters [48]- and known Auger transitions ( $\mathrm{Ar}, \mathrm{Kr}, \mathrm{Xe}$ [53]). The electron absolute detection efficiency with kinetic energy was determined through coincidences between $\mathrm{Kr} 3 \mathrm{~d}$ or $\mathrm{Ar} 2 \mathrm{p}$ photoelectrons at different photon energies, and Auger electrons: it is about $70 \%$ from 0 to $200 \mathrm{eV}$ and decreases slowly to about $40 \%$ at $800 \mathrm{eV}$ (electron loss by the magnetic mirror). This efficiency allows a good determination of relative cross section between different processes and in particular between double core ionization and single core ionization since all the processes are observed simultaneously. The detection efficiency for 4 electrons detected in coincidence is about $20 \%$. The relative energy resolution $\Delta \mathrm{E} / \mathrm{E}$ is typically $1.5 \%$ to $2 \%$.

\section{$\underline{\text { Results and discussion. }}$}

\section{$1-\mathrm{K}^{-2}$ double core ionization}

Our first experimental proof of $\mathrm{K}^{-2}$ double $\mathrm{K}$-shell ionization was done at Photon Factory on $\mathrm{N}_{2}$ at a photon energy of $1110 \mathrm{eV}$ and at SOLEIL on oxygen compounds: $\mathrm{O}_{2}, \mathrm{CO}$ and $\mathrm{CO}_{2}$ at an energy of $1300 \mathrm{eV}$ allowing $\mathrm{K}^{-2}$ ionization of the oxygen K-shell [4]. Comparable experiments were performed at the same time by Feifel's group at BESSY on $\mathrm{CH}_{4}$ and $\mathrm{NH}_{3}$ [5]. A recent review of these results was recently published [54].

For all these molecules (noted $A B$ below) the excitation and decay processes are the following (1):

$\mathrm{AB}+h v \rightarrow \mathrm{AB}^{2+}\left(\mathrm{K}^{-2}\right)+2 \mathrm{e}^{-}{ }_{1,2} \quad\left(\mathrm{KEe}_{1}, \mathrm{KEe}_{2}\right), \quad \mathrm{K}^{-2}$ double core ionization

$\mathrm{AB}^{2+}\left(\mathrm{K}^{-2}\right) \rightarrow \mathrm{AB}^{3+}\left(\mathrm{K}^{-1} \mathrm{~V}^{-2}\right)+\mathrm{e}_{\mathrm{A} 1}^{-}\left(\mathrm{KEe}_{\mathrm{A} 1}\right)$, 1st step hypersatellite Auger decay

$A B^{3+}\left(K^{-1} V^{-2}\right) \rightarrow A B^{4+}\left(V^{-4}\right)+e^{-}{ }^{-1} \quad\left(K E e_{A 2}\right), \quad$ 2nd step Auger decay

In our experiments, we have selected 4-electron coincidence events: the Hypersatellite Auger electron, the slower second Auger electron and the two photoelectrons that share the available excess energy: $\mathrm{KEe}_{1}+\mathrm{KEe}_{2}=\mathrm{h} v$ - (Binding Energy of $\mathrm{K}^{-2}$ states). The energy difference between the mean energy of hypersatellite Auger electrons and mean energy of the second Auger electrons is about $60-80 \mathrm{eV}$ (increasing from $\mathrm{C}$ to $\mathrm{N}$ and $\mathrm{O}$ ), the corresponding difference in time-of-flight of these two Auger electrons is longer than the dead time of our system and only a few coincidences are lost when the energy difference between the Auger electrons is less than 20-30 eV (from C to $\mathrm{N}$ and $\mathrm{O}$ ).

The detection in coincidence of four electrons reduces the background signal but detecting in coincidence the two photoelectrons with only the hypersatellite Auger electron gives also good results for $\mathrm{K}^{-2}$ spectroscopy [5].

For the nitrogen molecule [4], the energy correlation between the two photoelectrons is plotted in Fig 1a and the energy spectrum of $\mathrm{K}^{-2}$ states deduced from $\mathrm{hv}-\left(\mathrm{KEe}_{1}+\mathrm{KEe}_{2}\right)$ is shown in Fig $1 \mathrm{~b}$ for nitrogen. In addition to the first peak corresponding to the $\mathrm{K}^{-2}$ ground state, satellite peaks appear at higher binding energy resulting from simultaneous excitation of a valence electron to an unoccupied orbital. The contribution of $\mathrm{K}^{-2}$ satellite states is about $25 \%$ of the $\mathrm{K}^{-2}$ ground state, much more important than in $\mathrm{K}^{-1}$ satellites in single ionization (less than $5-10 \%$ of the main $\mathrm{K}^{-1}$ line). The perturbation of the valence electrons by the removal of two $1 \mathrm{~s}$ electrons induces here a stronger shake-up of the valence electron.

The Auger electron spectrum following the formation of the $\mathrm{K}^{-2}$ ground states is shown in fig 1c. The hypersatellite energy region [375-430eV] is clearly separated from the second Auger electron [300-375eV]. The 2D coincidence image between the Auger electrons 
(Fig 4 in ref [4]) gives complete information on the two-step Auger decay from $\mathrm{K}^{-2}$ ground state and also from the $\mathrm{K}^{-2}$ first satellite state where a high energy shoulder corresponding to participator Auger decay of the excited valence electron is also visible. However, due to the limited absolute resolution of the magnetic bottle for high kinetic energy electrons (about 6-8 eV for $\mathrm{N}_{2}$ Auger electrons) it is not possible to identify very precisely the intermediate $\mathrm{N}_{2}{ }^{3+}$ and $\mathrm{N}_{2}{ }^{4+}$ final states.
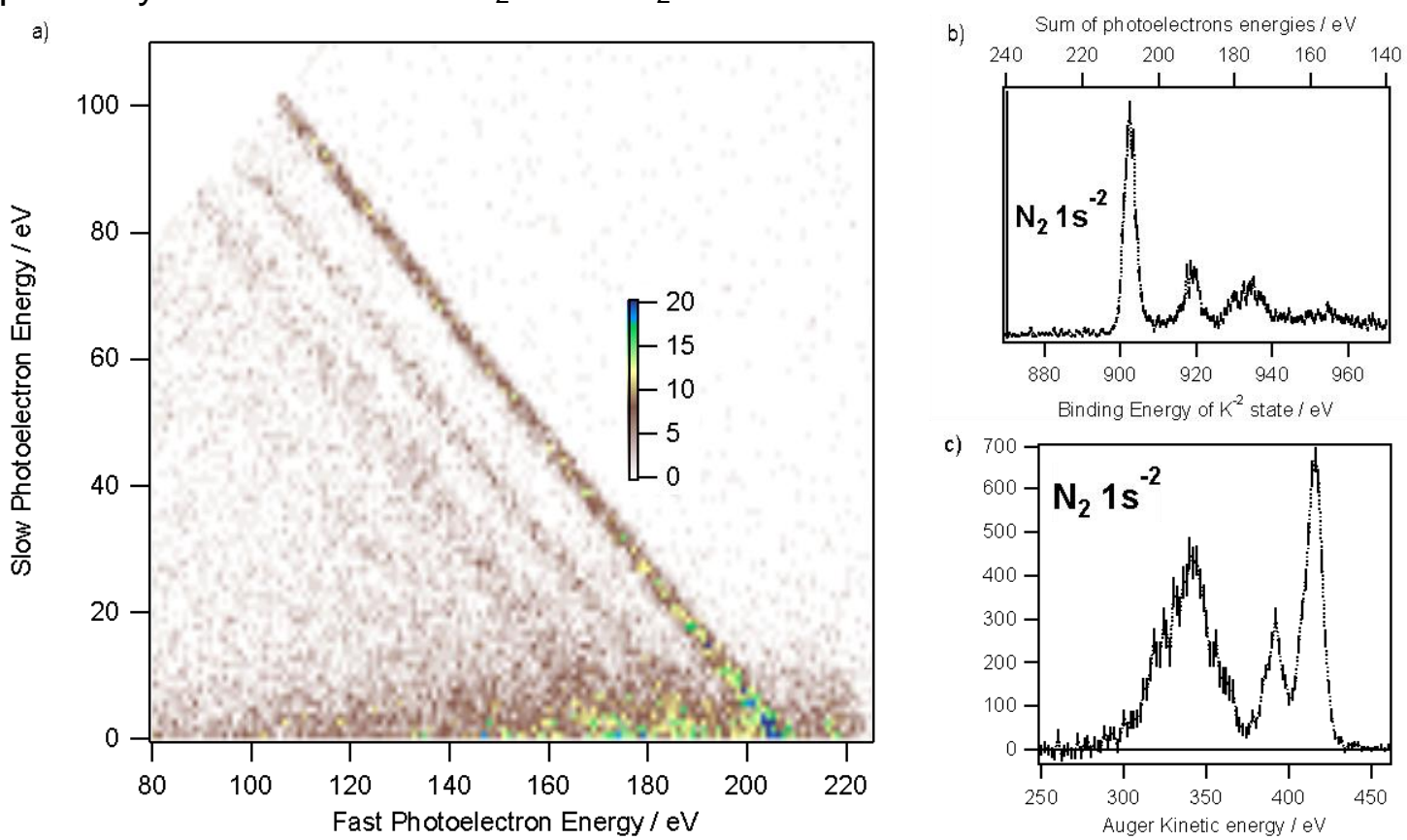

Figure 1: (a) Energy correlation between two photoelectrons emitted in $\mathrm{K}^{-2}$ double core ionization of $\mathrm{N}_{2}$ at $\mathrm{hv}=1110 \mathrm{eV}$, associated with two Auger electrons in the kinetic energy ranges of [375 $450 \mathrm{eV}]$ and [300 - $375 \mathrm{eV}]$. (b) Histogram of the sum of the energies of the two photoelectrons giving the spectrum of $\mathrm{K}^{-2}$ states. (c) Auger electrons energy distribution for the $\mathrm{K}^{-2}$ ground state.

Although a chemical shift is observed for $\mathrm{O}\left(\mathrm{K}^{-2}\right)$ double ionization between $\mathrm{O}_{2}, \mathrm{CO}$ and $\mathrm{CO}_{2}$ (Fig 3 in ref [4]), this shift is only a little bit more than for single K-shell ionization and is sensitive to the chemical environment in the same way. In these first results [4], the statistics was not good enough to observe $\mathrm{K}^{-1} \mathrm{~K}^{-1}$ ionization on two neighbor atoms in a molecule. This later process, owing to Cerderbaum's et al predictions [1] [2], must be sensitive to the bond length.

Hence, our next goal was the observation of the signature of this process in a given molecule and the comparison with the predictions of Cederbaum's along the $\mathrm{C}_{2} \mathrm{H}_{2} \mathrm{n}$ series [1].

2- $\mathrm{K}^{-1} \mathrm{~K}^{-1}$ double core ionization:

The acetylene molecule was our first choice to look for $\mathrm{K}^{-1} \mathrm{~K}^{-1}$ double ionization for a few reasons.

- The photon energy to ionize two K-shell electrons in $\mathrm{C}$ atom is lower than for $\mathrm{N}$ and $\mathrm{O}$ and the photon flux of the beamlines at PF and SOLEIL is higher around $\sim 700 \mathrm{eV}$ than above $\sim 1000 \mathrm{eV}$ (N atom) or $\sim 1300 \mathrm{eV}$ (O atom).

- The 1s cross section is also higher for $\mathrm{C}$ than for $\mathrm{N}$ and $\mathrm{O}$ and since the kinetic energy of the Auger from carbon is also lower, the collection efficiency of the magnetic bottle for Auger electrons is better.

-The triple bond length in acetylene is only $1.2 \AA$ and because the process for $\mathrm{K}^{-1} \mathrm{~K}^{-1}$ double ionization should be knock-out, it is expected to be more efficient in this molecule.

The process for $\mathrm{K}^{-1} \mathrm{~K}^{-1}$ double ionization and further decay are the following: 


$$
\begin{array}{lll}
\mathrm{C}_{2} \mathrm{H}_{2}+h \nu \rightarrow \mathrm{C}_{2} \mathrm{H}_{2}{ }^{2+}\left(\mathrm{K}^{-1} \mathrm{~K}^{-1}\right)+2 \mathrm{e}^{-} & \left(\mathrm{KEe}_{1}, \mathrm{KEe}_{2}\right), \quad \mathrm{K}^{-1} \mathrm{~K}^{-1} \text { double core ionization } \\
\mathrm{C}_{2} \mathrm{H}_{2}{ }^{2+}\left(\mathrm{K}^{-1} \mathrm{~K}^{-1}\right) \rightarrow \mathrm{C}_{2} \mathrm{H}_{2}{ }^{3+}\left(\mathrm{K}^{-1} \mathrm{~V}^{-2}\right)+\mathrm{e}^{-}{ }_{\mathrm{A} 1} & \left(\mathrm{KEe}_{\mathrm{A} 1}[220-250 \mathrm{eV}]\right), 1^{\text {st }} \text { step Auger decay } \\
\mathrm{C}_{2} \mathrm{H}_{2}{ }^{3+}\left(\mathrm{K}^{-1} \mathrm{~V}^{-2}\right) \rightarrow \mathrm{C}_{2} \mathrm{H}_{2}{ }^{4+}\left(\mathrm{V}^{-4}\right)+\mathrm{e}_{\mathrm{A} 2}^{-} & \left(\mathrm{KEe}_{\mathrm{A} 2}[220-250 \mathrm{eV}]\right), 2^{\text {nd }} \text { step Auger decay }
\end{array}
$$

The experimental difficulty to extract the corresponding $\mathrm{K}^{-1} \mathrm{~K}^{-1}$ signal is due to the fact that the signal is much weaker than for $\mathrm{K}^{-2}$ and that the two Auger electrons are in the same energy range [215-260eV]. It was however possible (after $12 \mathrm{~h}$ of accumulation at $20 \mathrm{KHz}$ total electron count rate) to evidence the $\mathrm{K}^{-1} \mathrm{~K}^{-1}$ peak at a binding energy of $595.6 \pm 0.5 \mathrm{eV}$ by detecting only one of the two Auger electrons in coincidence with two photoelectrons [8]. Knowing electron detection efficiencies, it was possible to determine a ratio of $\mathrm{K}^{-1} \mathrm{~K}^{-1}$ to $\mathrm{K}^{-2}$ cross-section of $2 \%$ and $\mathrm{K}^{-1} \mathrm{~K}^{-1}$ to $\mathrm{K}^{-1}$ of $2.10^{-5}$. This shows the sensitivity of the method to extract such minor processes.

In subsequent experiments, it was also possible to evidence the $\mathrm{K}^{-1} \mathrm{~K}^{-1}$ peaks in $\mathrm{C}_{2} \mathrm{H}_{4}$, $\mathrm{C}_{2} \mathrm{H}_{6}, \mathrm{~N}_{2}$ and $\mathrm{CO}$ [9]. The corresponding binding energies were found at 593.3 $\pm 0.5 \mathrm{eV}$ $\left(\mathrm{C}_{2} \mathrm{H}_{4}\right), 590.2 \pm 0.5 \mathrm{eV}\left(\mathrm{C}_{2} \mathrm{H}_{6}\right), 835.9 \pm 1 \mathrm{eV}\left(\mathrm{N}_{2}\right)$ and 855.4 $\pm 1 \mathrm{eV}(\mathrm{CO})$.
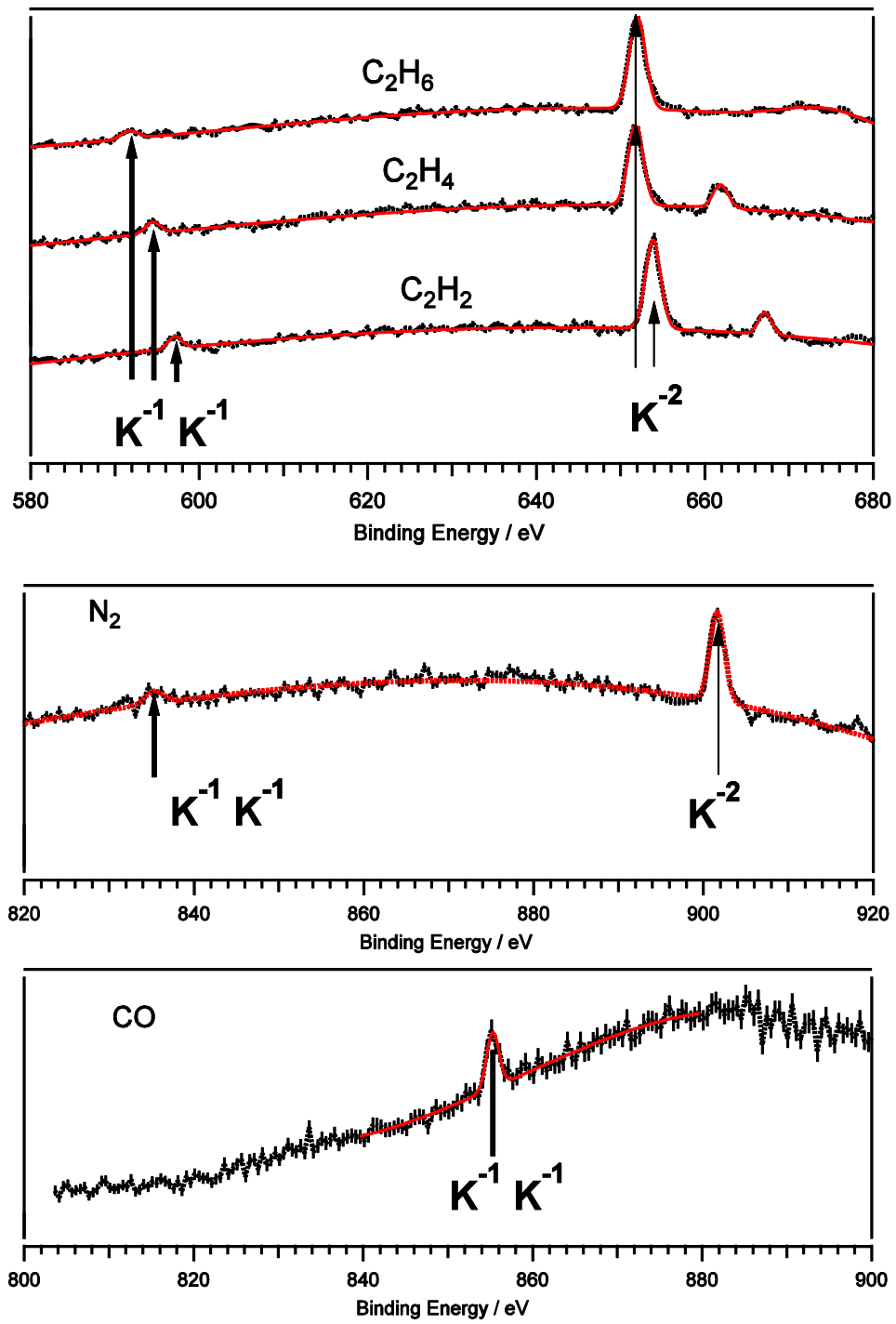

Figure 2: Evidence of the $\mathrm{K}^{-1} \mathrm{~K}^{-1}$ signal for $\mathrm{C}_{2} \mathrm{H}_{2}, \mathrm{C}_{2} \mathrm{H}_{4}, \mathrm{C}_{2} \mathrm{H}_{6}$ at $\mathrm{h} v=770 \mathrm{eV}, \mathrm{N}_{2}$ at $\mathrm{hv}=1005 \mathrm{eV}$ and $\mathrm{CO}$ at $\mathrm{h} v=953 \mathrm{eV}$. The red lines show the fit of our experimental data for the $\mathrm{K}^{-1} \mathrm{~K}^{-1}$ and $\mathrm{K}^{-2}$ peaks by Gaussian functions and a cubic background.

Fig 2 shows the corresponding spectra that were first published in ref. [9] and clearly reveal the strong chemical shift for $\mathrm{K}^{-1} \mathrm{~K}^{-1}$ along the $\mathrm{C}_{2} \mathrm{H}_{2 n}$ series. These experimental values are in good agreement with theoretical calculations [9] [27] and confirm 
Cederbaum et al's prediction [1]. The chemical shift depends on the bond length in the $\mathrm{C}_{2} \mathrm{H}_{2 n}(\mathrm{n}=1-3)$ series and involves both Coulomb interaction between the two $1 \mathrm{~s}$ holes and relaxation energies. The $\mathrm{K}^{-1} \mathrm{~K}^{-1}$ cross-section decreases as $1 / \mathrm{R}^{2}$ with the $\mathrm{C}-\mathrm{C}$ bond length [9] and validates the knockout process where a 1s electron ejected from the first carbon atom ejects by internal collision another $1 \mathrm{~s}$ electron from the neighbor atom. The ratio of the $\mathrm{K}^{-1} \mathrm{~K}^{-1} / \mathrm{K}^{-2}$ ionization cross sections are of the order of $1-2 \%$ and the ratio of $\mathrm{K}^{-1} \mathrm{~K}^{-1} / \mathrm{K}^{-1}$ ionization cross sections about $10^{-5}$ [9].

It was also possible to plot the 2D coincidences between the two Auger electrons in the $\mathrm{K}^{-2}$ decay of $\mathrm{C}_{2} \mathrm{H}_{2 \mathrm{n}}(\mathrm{n}=1-3)$ [55] and to compare with theoretical calculations [56].

In further experiments, the dead time was reduced to 6ns (imposed by our TDC) by careful settings of the MCP signal and we succeeded in detecting 4 electrons in coincidence also for the $\mathrm{K}^{-1} \mathrm{~K}^{-1}$ process. With a 6 ns dead time, when two Auger electrons are selected in the energy window [215-270 eV], it is possible to find one faster than the other by $\sim 12 \mathrm{eV}$ and this allows the detection of a second Auger electron 6ns later. Even if a lot of the signal is lost due to this dead time, and forbids any estimation of the relative cross section, the signal to noise becomes better because the background due to random coincidences is much reduced. For $\mathrm{C}_{2} \mathrm{H}_{4}$, the corresponding spectrum, collected at $770 \mathrm{eV}$ photon energy, is shown in the upper panel of Fig. 3 and the $\mathrm{K}^{-2}$ signal in the lower panel. The $\mathrm{K}^{-1} \mathrm{~K}^{-1}$ peak is found at $593 \pm 1 \mathrm{eV}$ in good agreement with our published results [9]. We notice that the $\mathrm{K}^{-2}$ signal appears also when the two Auger electrons are taken in the same [215-260 eV] energy window. This means that some hypersatellite Auger electrons are also present in this region but this contribution is only of the order of $1 \%$.

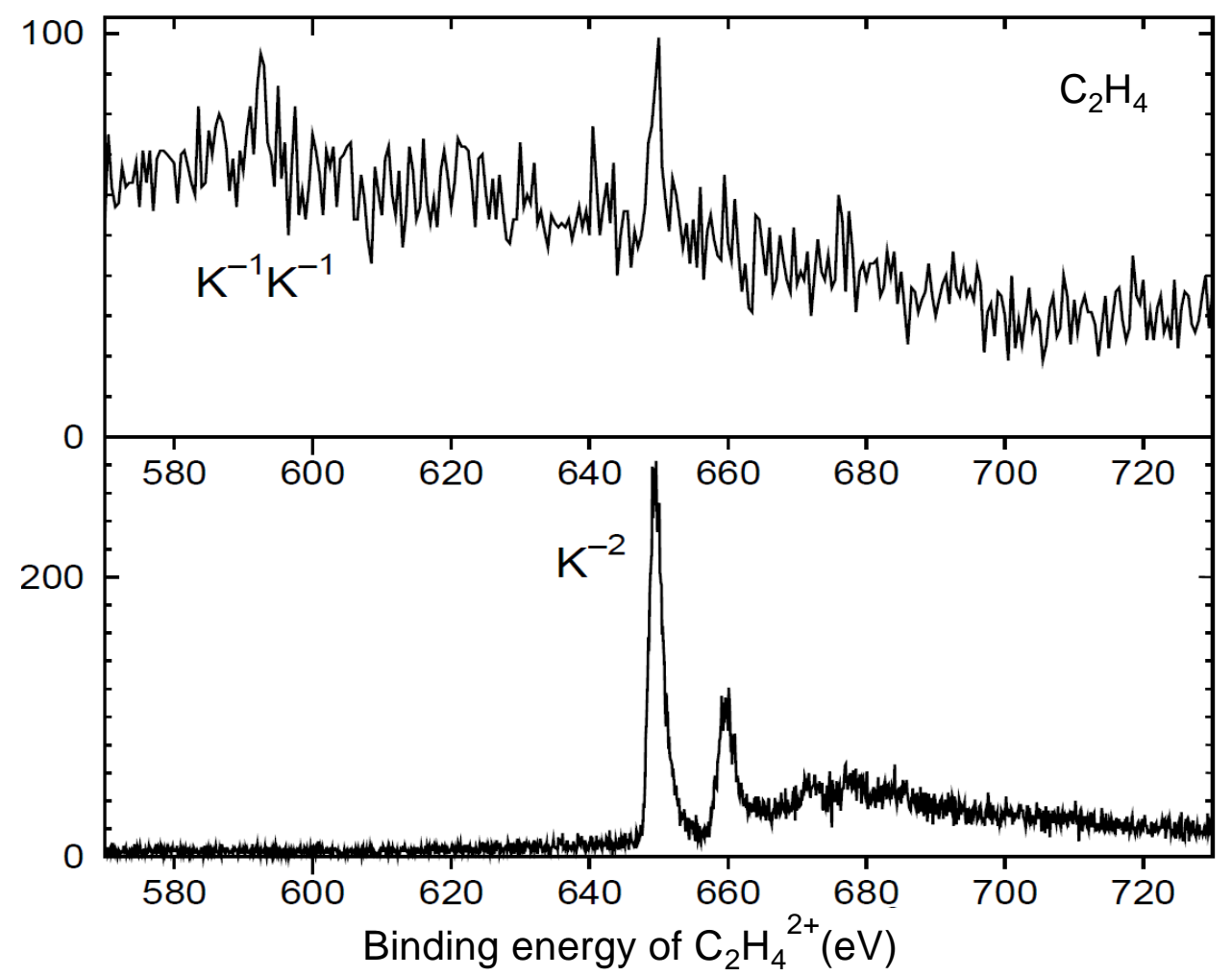

Figure 3. Upper panel: $\mathrm{K}^{-1} \mathrm{~K}^{-1}$ Double ionization signal deduced from 4 electron coincidences (2 photoelectrons and 2 Auger electrons in [215-260 eV] energy range). $B E=h v-\left(K_{E e}+K_{1 E e_{2}}\right)$. Lower panel: $\mathrm{K}^{-2}$ Double ionization signal deduced from 4 electron coincidences (2 photelectrons 
and 2 Auger electrons in [215-270 eV] and [270-315 eV] energy ranges. The photon energy is $770 \mathrm{eV}$.

\section{New results:}

\section{Propylene:}

The significant energy shift observed for $\mathrm{K}^{-1} \mathrm{~K}^{-1}$ double ionization in the $\mathrm{C}_{2} \mathrm{H}_{2 n}$ [9] series has led us to study if the same effect could be observed inside the same molecule. We have performed studies in benzene $\left(\mathrm{C}_{6} \mathrm{H}_{6}\right)$, propyne and propadiene $\left(\mathrm{C}_{3} \mathrm{H}_{4}\right)$, and propylene $\left(\mathrm{C}_{3} \mathrm{H}_{6}\right)$. The results for $\mathrm{C}_{6} \mathrm{H}_{6}$ and $\mathrm{C}_{3} \mathrm{H}_{4}$ are under analysis and will be published later. We show here the results for propylene: $\mathrm{H}_{3} \mathrm{C}^{1}-\mathrm{C}^{2} \mathrm{H}=\mathrm{C}^{3} \mathrm{H}_{2}\left(\mathrm{C}^{\mathrm{i}}\right.$ notations are used to differentiate the three different carbon atoms). The single and double bonds with respective length of 1.5 and $1.34 \AA$ are similar to those of $\mathrm{C}_{2} \mathrm{H}_{6}$ and $\mathrm{C}_{2} \mathrm{H}_{4}$. So that, if the energy was only due to $\mathrm{K}^{-1} \mathrm{~K}^{-1}$ coulomb interaction (neglecting inductive effects and relaxation), an energy shift of about $3 \mathrm{eV}$ (as observed between $\mathrm{C}_{2} \mathrm{H}_{6}$ and $\mathrm{C}_{2} \mathrm{H}_{4}$ ) could be expected between $\mathrm{K}^{-1} \mathrm{~K}^{-1}$ ionization on $\mathrm{C}^{1}-\mathrm{C}^{2}$ and $\mathrm{C}^{2}=\mathrm{C}^{3}$ in propylene. Due to the knockout process that is the main responsible for $\mathrm{K}^{-1} \mathrm{~K}^{-1}$ formation [9] there is very little chance to observe a third peak corresponding to $\mathrm{K}^{-1} \mathrm{~K}^{-1}$ double ionization on $\mathrm{C}^{1}$ and $\mathrm{C}^{3}$.

The data for propylene were acquired in the same conditions than for $\mathrm{C}_{2} \mathrm{H}_{4}$ at photon energies of 710 and $770 \mathrm{eV}$.

By filtering out 4 electron events with two photoelectrons, one hypersatellite Auger electron in the [280-315 eV] energy range and a second Auger in the [215-280 eV] energy range it was possible to plot the 2D coincidence map between the two photoelectrons (Fig 4) and to deduce the $\mathrm{K}^{-2}$ spectrum in the lower right panel of Fig 4.
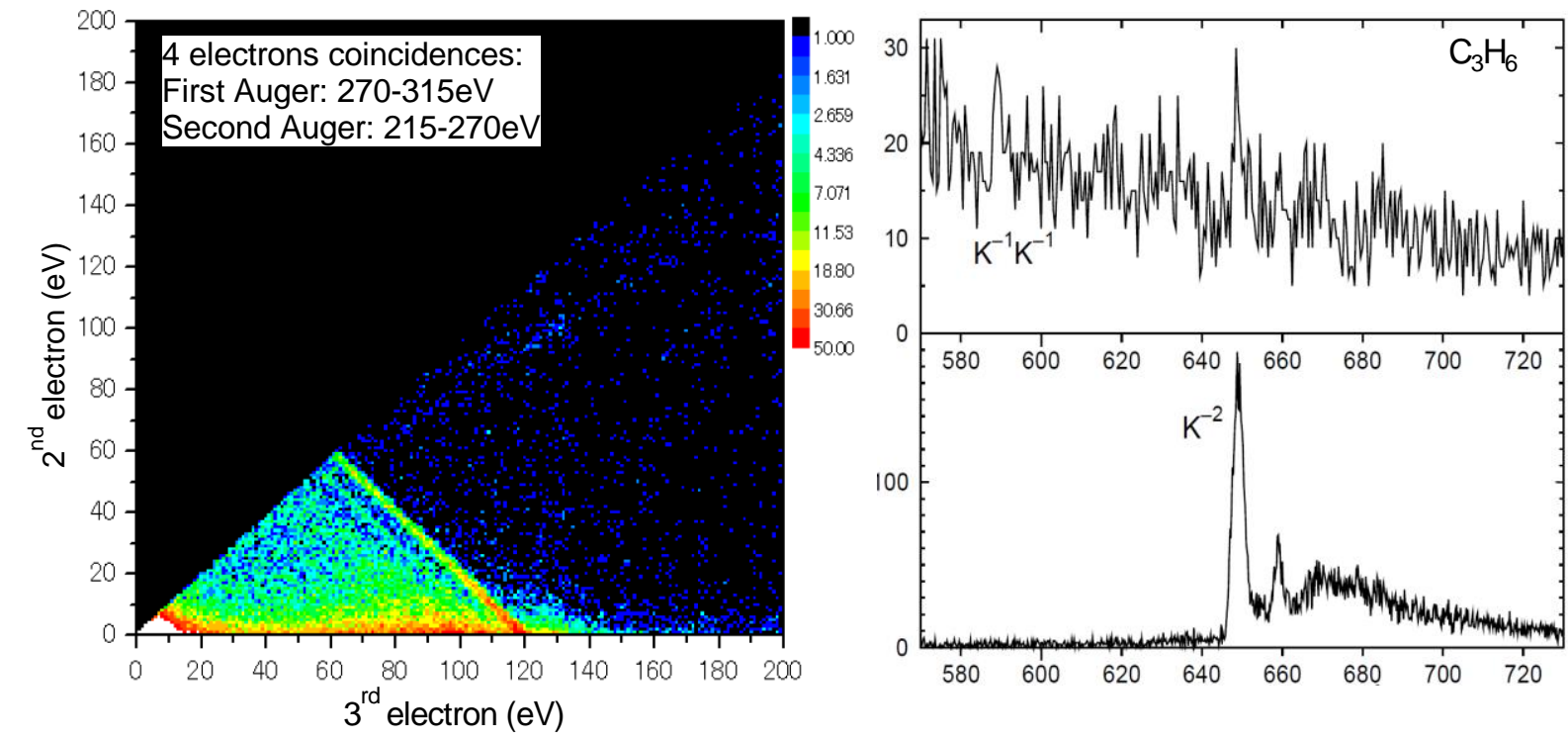

Fig 4: left: Energy correlation between two photoelectrons from $\mathrm{C}_{3} \mathrm{H}_{6}$ measured at $\mathrm{h} v=770 \mathrm{eV}$; right, lower panel: $\mathrm{K}^{-2}$ signal; right, upper panel: $\mathrm{K}^{-1} \mathrm{~K}^{-1}$ signal

As it has been shown for $\mathrm{C}_{2} \mathrm{H}_{4}$ in the previous paragraph, it has been also possible to select 4-electron events with two Auger electrons in the same energy range [215-270eV]. The $\mathrm{K}^{-1} \mathrm{~K}^{-1}$ peak is clearly visible in Fig 4 (upper panel, right) with a weak $\mathrm{K}^{-2}$ signal filtered in these conditions (see above).

For propylene, the energy difference between $\mathrm{K}^{-2}$ and $\mathrm{K}^{-1} \mathrm{~K}^{-1}$ peaks is $59.5 \pm 0.5 \mathrm{eV}$ setting the $\mathrm{K}^{-1} \mathrm{~K}^{-1}$ level at $591 \mathrm{eV}$ at an intermediate value between $\mathrm{C}_{2} \mathrm{H}_{6}$ and $\mathrm{C}_{2} \mathrm{H}_{4}$ [9]. Unfortunately the resolution around the $\mathrm{K}^{-1} \mathrm{~K}^{-1}$ peak $(180 \mathrm{eV}$ kinetic energy sum of the two electrons) of about $5 \mathrm{eV}$ is not sufficient to identify two peaks in the $\mathrm{K}^{-1} \mathrm{~K}^{-1}$ signal with the statistics we have. As in all other systems [9], above the $\mathrm{K}^{-1} \mathrm{~K}^{-1}$ peak we cannot 
distinguish any satellite structure. Although the statistics is not very good, it could be sufficient to observe a structure higher than $20 \%$ of the main peak. We think that $\mathrm{K}^{-1} \mathrm{~K}^{-1}$ satellite relative intensities with respect to $\mathrm{K}^{-1} \mathrm{~K}^{-1}$ are closer to the ratio of the satellite of the single $\mathrm{K}^{-1}$ line (less than 10\%) because the local perturbation is smaller when the two holes are located on neighbor atoms.

If we compare the $\mathrm{K}^{-2}$ signal for propylene and ethylene (Fig 3 and 4 ) we can see a clear broadening of the main $\mathrm{K}^{-2}$ peak that indicates that some chemical shift exist for $\mathrm{K}^{-2}$ ionization on the three different carbon atoms. Even if it is not possible to isolate three separated threshold, this broadening is definitely larger than the $\mathrm{K}^{-2}$ shift between $\mathrm{C}_{2} \mathrm{H}_{4}$ and $\mathrm{C}_{2} \mathrm{H}_{6}$ which is only $0.2 \mathrm{eV}$ [9]. Similar measurement at a photon energy of $710 \mathrm{eV}$ (Fig. $6 \mathrm{~b})$ provide a better energy resolution $(\sim 2 \mathrm{eV})$ and indicate that the $\mathrm{K}^{-2}$ peak is a composite of different contributions but the cross section is smaller at this photon energy and the different components are not yet resolved. The calculations using different theoretical model used in ref. [9]: SCF/HF; MP2 and DFT/B3LYP, find an energy difference $\Delta\left(\mathrm{K}^{-2}\left(\mathrm{C}^{2}\right)-\mathrm{K}^{-2}\left(\mathrm{C}^{1}\right)\right)=0.9 \pm 0.2 \mathrm{eV}$ and $\Delta\left(\mathrm{K}^{-2}\left(\mathrm{C}^{3}\right)-\mathrm{K}^{-2}\left(\mathrm{C}^{1}\right)\right)=2.3 \pm 0.3 \mathrm{eV}$ which is fully compatible with the observed broadening. Such difference in $\mathrm{K}^{-2}$ ionization energy for the three carbon atoms in $\mathrm{C}_{3} \mathrm{H}_{6}$ does not exist between $\mathrm{C}_{2} \mathrm{H}_{6}$ and $\mathrm{C}_{2} \mathrm{H}_{4}$ whereas, in a very naive picture, propylene could be seen as a "concatenation" of ethane and ethylene. This is due to different relaxation energies for the three different double core hole localization in $\mathrm{H}_{3} \mathrm{C}^{1}-\mathrm{C}^{2} \mathrm{H}=\mathrm{C}^{3} \mathrm{H}_{2}$ rather than to the Coulomb interaction. In a further step, it would be interesting to compare experimental results on propylene, propane, propyne and propadiene with theoretical calculations to understand the role of Coulomb interaction and relaxation energies [1] [27] [57] for all these hydrocarbon molecules with 3 carbon atoms.

\section{Ethyl trifluoroacetate:}

The ethyl trifluoroacetate molecule $\left(\mathrm{C}^{1} \mathrm{H}_{3} \mathrm{C}^{2} \mathrm{H}_{2} \mathrm{OC}^{3} \mathrm{OC}^{4} \mathrm{~F}_{3}\right.$, the i exponent $\mathrm{C}^{\mathrm{i}}(\mathrm{i}=1-4)$ is used to identify the different $\mathrm{C}$ atoms) was the molecule chosen by Kai Siegbahn [3] [58] to demonstrate clearly the chemical shift for $\mathrm{K}^{-1}$ ionization on 4 carbon atoms with very different environment. In a previous study [59] we could isolate the specific Auger decay of each carbon atom in this molecule. Here, our goal was to observe the double K-shell ionization on different carbon sites and, if possible, $\mathrm{K}^{-1} \mathrm{~K}^{-1}$ on neighbor carbon atoms.

The single K-shell photoelectron spectra observed at a photon energy of $320 \mathrm{eV}$ is shown in Fig 5c. The photon energy to observe carbon $\mathrm{K}^{-2}$ process was set to $740 \mathrm{eV}$. However, at this photon energy the $\mathrm{O}$ and $\mathrm{F} \mathrm{K}$-shell ionization channels are opened and are responsible for most of the electron signal. The 2D coincidence map in fig 5 a shows the coincidences between 2 photoelectrons when two Auger electrons from carbon are also detected (4-fold coincidences). The diagonal lines correspond to the expected $\mathrm{C}\left(\mathrm{K}^{-2}\right)$ processes. However an intense vertical line is also observed that corresponds to Fluorine K-shell photoelectrons. This corresponds to random coincidences in 4-fold coincidence involving a $\mathrm{F}\left(\mathrm{K}^{-1}\right)$ photoelectron from another molecule with three electrons from $\mathrm{C}\left(\mathrm{K}^{-2}\right)$ (2 Auger and 1 photoelectron). That could only be reduced (quadratically) by reducing the count rate. But doing this, the true $\mathrm{K}^{-2}$ coincidences count rate also decreases (linearly) and a much longer acquisition time would be necessary. Since the $\mathrm{K}^{-2}$ process is only about a $10^{-3}$ fraction of single $\mathrm{K}$-shell ionization, when the $\mathrm{O}$ and $\mathrm{F} \mathrm{K}$ shell photoelectrons are in the region of interest to observe $\mathrm{C}\left(\mathrm{K}^{-2}\right)$ ionization there is no other way than accumulating data for a very long time. The corresponding spectrum was accumulated during 12 hours at a count rate of $15 \mathrm{kHz}$. The final result in Fig $5 \mathrm{~b}$ ) reveals clearly the four $\mathrm{K}^{-2}$ peaks associated to the different carbon atoms even if the statistics is limited and the background higher than in previous experiments. Since $\mathrm{K}^{-2}$ satellite states are also probably present in this region with an energy shift of about $10 \mathrm{eV}$ with respect to the $\mathrm{K}^{-2}$ peak it is possible to have some overlap between $\mathrm{C}^{1}\left(\mathrm{CH}_{3}\right.$ termination) $\mathrm{K}^{-2}$ satellites and $\mathrm{C}^{4}\left(\mathrm{CF}_{3}\right.$ termination) $\mathrm{K}^{-2}$ ground state peaks. The experimental results are very well reproduced by theoretical calculation using different 
models in table 1 . Note that the observed $\mathrm{K}^{-2}$ chemical shifts are approximately twice the $\mathrm{K}^{-1}$ chemical shifts as reported by Travnikova et al [60]

\begin{tabular}{|l|l|l|l|l|l|}
\hline & $\begin{array}{c}\mathrm{K}^{-2} \mathrm{BE}(\mathrm{eV}) \\
\text { Exp. } \pm 1 \mathrm{eV}\end{array}$ & $\begin{array}{c}\mathrm{K}^{-2} \mathrm{BE}(\mathrm{eV}) \\
(\mathrm{SCF} / \mathrm{HF})\end{array}$ & $\begin{array}{c}\mathrm{K}^{-2} \mathrm{BE}(\mathrm{eV}) \\
(\mathrm{MP} 2)\end{array}$ & $\begin{array}{c}\mathrm{K}^{-2} \mathrm{BE}(\mathrm{eV}) \\
(\mathrm{DFT} / \mathrm{B} 3 \mathrm{LYP})\end{array}$ & $\begin{array}{c}\mathrm{K}^{-1}(\mathrm{eV}) \\
\text { Exp. }\end{array}$ \\
\hline $\mathrm{C}^{1}\left(\mathrm{CH}_{3}\right)$ & 651.7 & 650.48 & 651.32 & 649.50 & $291.47(0)$ \\
\hline $\mathrm{C}^{2}\left(\mathrm{CH}_{2}\right)$ & $653.7(2)$ & $653.17(2.69)$ & $653.88(2.56)$ & $651.64(2.14)$ & +1.72 \\
\hline $\mathrm{C}^{3}(\mathrm{CO})$ & $659.2(7.5)$ & $660.45(9.97)$ & $659.53(8.21)$ & $656.61(7.11)$ & +4.33 \\
\hline $\mathrm{C}^{4}\left(\mathrm{CF}_{3}\right)$ & $666.3(14.5)$ & $666.23(15.75)$ & $\begin{array}{l}665.95 \\
(14.63)\end{array}$ & $663.30(13.80)$ & +7.46 \\
\hline
\end{tabular}

Table 1: Comparison of experimental $\mathrm{K}^{-2}$ double core hole binding energies (BE) with different theoretical calculations. The experimental uncertainty is $\pm 1 \mathrm{eV}$. The relative energy shift for the different carbon localization with respect to $\mathrm{C}^{1}\left(\mathrm{CH}_{3}\right.$ termination) are indicated in parenthesis. $\mathrm{K}^{-1}$ chemical shifts are from the experiment by Travnikova et al $\mathrm{K}^{-1}$ chemical shifts are from the experiment by Travnikova et al [60].

For this molecule, with such limitations, it is hopeless to observe $\mathrm{K}^{-1} \mathrm{~K}^{-1}$ ionization that should represent about $1 \%$ of the $\mathrm{K}^{-2}$ process.

This shows the limits of the method that cannot be improved by any way since counts rates are defined by the ratio of double to single ionization cross-sections. It is in this field that multiphoton processes accessible with X-FEL intensities could bring a decisive advantage if coincidence experiments become possible in a reasonable time.

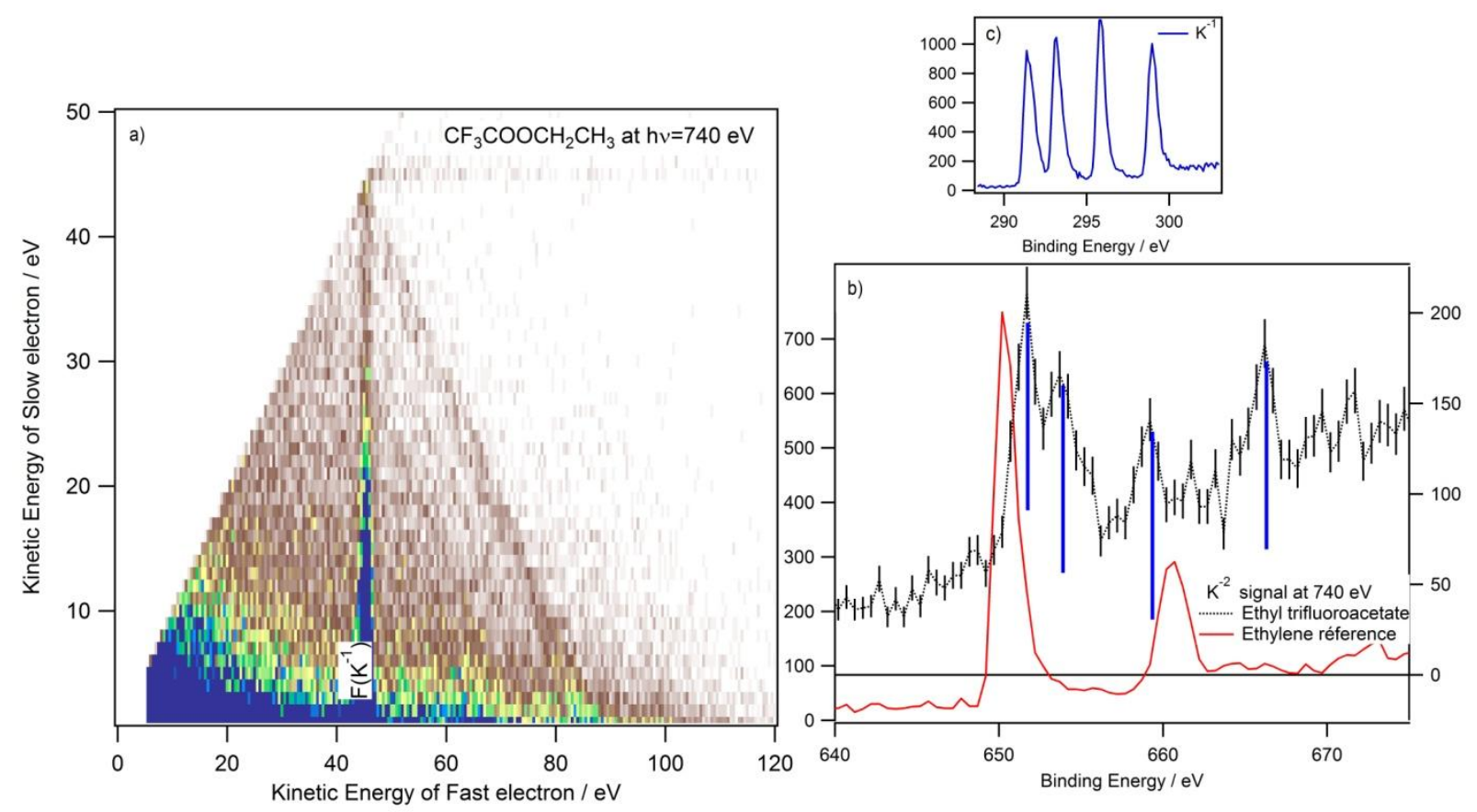

Figure 5: ethyl trifluoroacetate molecule a) 2D coincidence map between 2 photoelectrons (with 2 Auger electrons) measured at $\mathrm{h} v=740 \mathrm{eV}$, the line at $\sim 45 \mathrm{eV}$ corresponds to fluorine $\mathrm{K}$-shell photoelectrons and random coincidences. b) $\mathrm{K}^{-2}$ spectrum obtained by projection of the 2D map $\left(\mathrm{F}\left(\mathrm{K}^{-1}\right)\right.$ random coincidences were rejected), the $\mathrm{C}_{2} \mathrm{H}_{4} \mathrm{~K}^{-2}$ spectrum is used as a reference. $\left.\mathrm{c}\right) \mathrm{K}^{-1}$ spectrum at $h v=320 \mathrm{eV}$.

$\mathrm{K}^{-2} \mathrm{~V}$ spectroscopy:

In addition to double core hole ionization, related processes present also a high experimental and theoretical interest. These processes are the precursors of double core ionization and correspond to core ionization with simultaneous excitation of a core to a 
vacant orbital (shortly $\mathrm{K}^{-2} \mathrm{~V}$ ). Since single photon double core ionization is due to electron correlation, this process can be seen as aborted double K-shell ionization process.

In a recent publication [10], we have shown that this process has very interesting properties for the $\mathrm{C}_{2} \mathrm{H}_{2 n}$ series. States with different symmetries are observed in the spectra and reveal typical NEXAFS signatures. The theoretical model that was developed to describe this process has been detailed and applied to $\mathrm{N}_{2}$ and $\mathrm{H}_{2} \mathrm{O}$ molecules [47] [48]. The process can also be interpreted as a "super"-shake-up process following K-shell ionization. The interesting point is that the conjugate shake-up process that is rather weak in case of "normal" shake-up of valence electrons is, in the present case, comparable to the direct shake-up process of the remaining K-shell electron.

In a two-step image of the process, we have two pathways leading to such $\mathrm{K}^{-2} \mathrm{~V}$ states: 1- dipolar ionization of a first K-shell electron followed by monopolar shake-up transition of the remaining $\mathrm{K}$-shell electron to a vacant orbital, 2- dipole excitation of a first K-shell electron to a vacant orbital followed by monopolar shake-off of the remaining one.

It is interesting to show here this process in propylene molecule. To extract the $\mathrm{K}^{-2} \mathrm{~V}$ signal, three electron coincidence events with two Auger and only one photoelectron are selected to obtain the spectra presented in figure 6 . The photoelectron spectra is directly converted in binding energy $B E=h v-K E\left(e^{-} p h\right)$.

The spectrum in Fig 6 is very similar to what would be observed by summing up the equivalent spectra in $\mathrm{C}_{2} \mathrm{H}_{6}$ and $\mathrm{C}_{2} \mathrm{H}_{4}$ (Fig 2 in ref. [10]). The first peak at 635eV can hence be clearly assigned to the excitation of the $\pi^{*}$ resonance in the conjugate shakeup process and is related to the $\mathrm{C}^{2}=\mathrm{C}^{3}$ double bond. The next peak is more difficult to interpret due to three possible localizations of the double core vacancy with shake-up excitation of a K-shell electron into the $\sigma^{*}$ orbital.
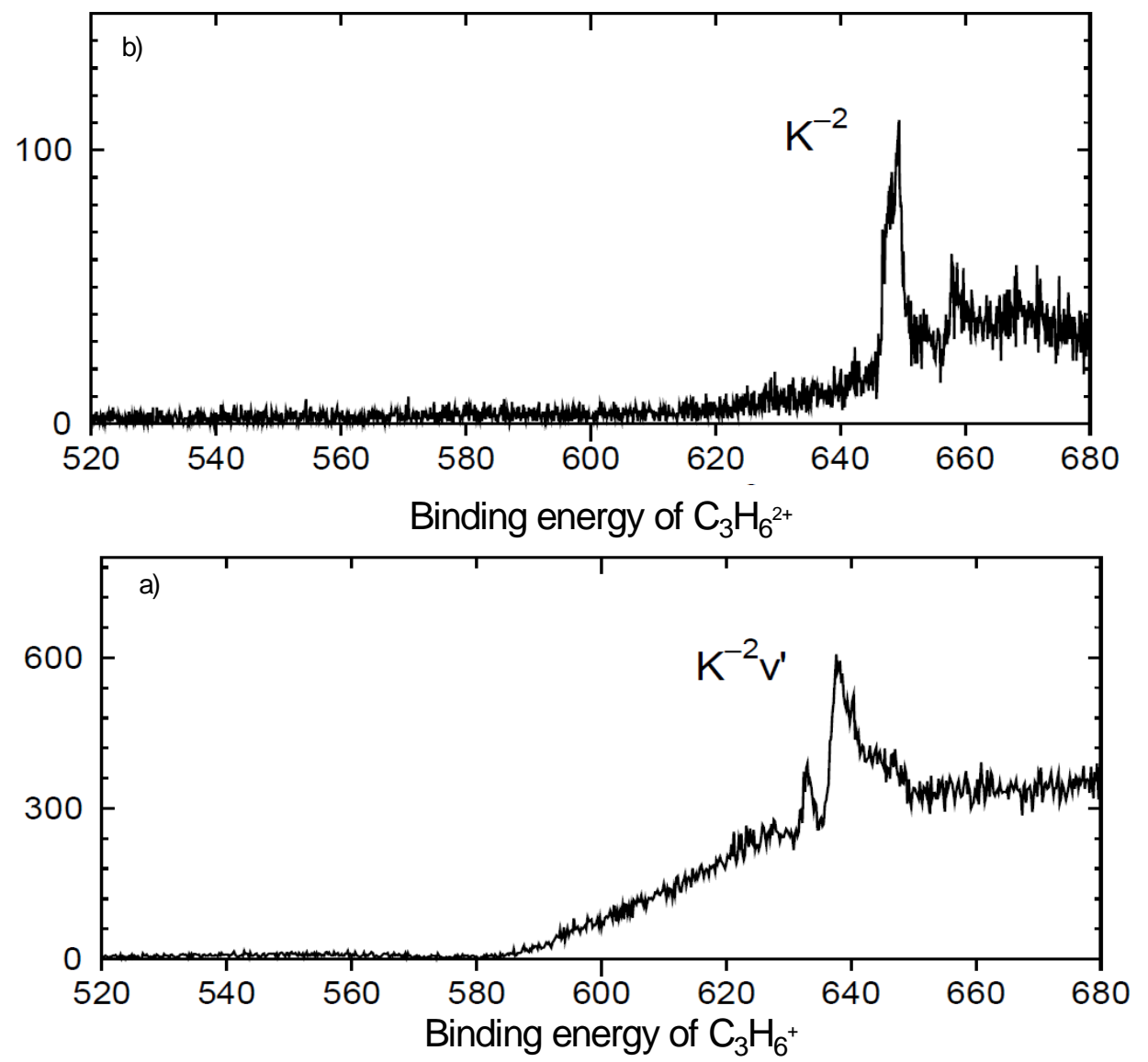

Figure 6. a) Propylene $\mathrm{K}^{-2} \mathrm{~V}$ spectrum obtained by detecting one photoelectron in coincidence with two Auger electrons at a photon energy of $710 \mathrm{eV}$. The background is due to other processes that 
have not been subtracted [47] [48]. The first peak at $635 \mathrm{eV}$ is due to $\pi^{*}$ resonance. b) $\mathrm{K}^{-2}$ spectrum at $h v=710 \mathrm{eV}$ showing the composite structure of the main peak due to the 3 different $\mathrm{C}$ atoms.

The spectroscopy of $\mathrm{K}^{-2} \mathrm{~V}$ states may appear richer than $\mathrm{K}^{-2}$ because states of different symmetries are observed with comparable intensities. Another strong experimental interest is that, since only one photoelectron is emitted, their study can be accessed by "normal" photoelectron spectroscopy without coincidence detection of the photoelectron with the two Auger electrons. Coincidences are useful in order to remove any ambiguity, in particular in the Auger spectra, when the overlap of hypersatellite Auger lines from $\mathrm{K}^{-2}$ states and $\mathrm{K}^{-2} \mathrm{~V}$ states forbids using this spectrum as a unique signature of the $\mathrm{K}^{-2}$ ionization process.

By choosing the photon energy to avoid any overlap between $\mathrm{K}^{-2} \mathrm{~V}$ photoelectrons and any other electron (photo electron or Auger electron) it is possible to obtain spectra with a high resolution electron spectrometer. The signal is about $10^{-3}$ fraction of the single $\mathrm{K}$ shell ionization but is well separated (by the K-shell excitation energy) from the corresponding photoelectron and "normal" satellite lines.

Such studies have been recently performed in sulfur containing molecules $\left(\mathrm{SF}_{6}, \mathrm{CS}_{2}\right)$ [61] on the Galaxies beamline at SOLEIL.

An additional interest of such measurements is the variation of the ratio between shakeup and conjugate shake-up with the photon energy. This variation was observed in a limited energy range in the case of $\mathrm{N}_{2}$ [48] but it was not possible to reach conditions close to the sudden approximation where only the direct shake-up line should subsist. This variation can be observed by varying the photon energy from $\mathrm{K}^{-2}$ threshold to up to $10 \mathrm{keV}$. A very simple image of the relative evolution of shake-up and conjugate shakeup states is given by the uncertainty principle through the energy detuning. If we consider the ionization of the K-shell electron, the shake-up of the other K-shell electron occurs in a timescale $\tau_{1}=\hbar / E_{K V}$ determined by the energy $E_{K V}$ that the photoelectron must give to excite the other $\mathrm{K}$-shell electron to a vacant orbital. On the other hand, the conjugate shake-up process implies first the excitation of a K-shell electron to the $\mathrm{V}$ orbital and the energy excess $h v-E_{K V}$ is given to shake off the other K-shell electron. The corresponding time, according to the uncertainty principle, to exchange energy between two electrons in this process is $\tau_{2}=\hbar /\left(h v-E_{K V}\right)$. Close to the $K^{-2}$ threshold, the two processes have similar intensities (because the photon energy is close to $2 \mathrm{E}_{\mathrm{KV}}$ ) but when the photon energy increases the ratio of conjugate to direct process evolves as $\tau_{2} / \tau_{1}$ and decreases when the photon energy increases.

It would be very interesting to verify quantitatively this simple image by recording the specific photoelectron signal at different photon energies.

\section{Conclusions:}

Double core hole spectroscopy has been recently utilized at two complementary light sources; synchrotrons and X-FEL light sources using different experimental approaches. The impressive collection of results in a few years has stimulated theoretical models and has confirmed the "prophetic" calculation by Cederbaum et al. [1] [2].

However, from a practical point of view these complementary methodologies remain technically difficult and will need further improvements to go beyond the present status. With synchrotron sources, the coincidence technique is able to provide high resolution spectroscopy of $\mathrm{DCH}$ states and this can be easily extended to $\mathrm{K}^{-2} \mathrm{~V}$ spectroscopy with up-to-date electron spectrometers and synchrotron beamlines. The real limitation comes from the very small cross-section of the order of $10^{-3}$ with respect to single K-shell ionization.

With X-FEL light sources, the cross section is not an issue but new experimental developments will be needed to fully disentangle all the possible processes. With much 
higher repetition rates $(27 \mathrm{kHz}$ burst mode at European X-FEL) one should certainly consider the possibility of coincidence spectroscopy to identify clearly all the possible channels. One could also consider a possible improvement of the "focus/unfocused" method used in refs. [23] and [26] that would consist of recording simultaneously the spectra with two identical spectrometers located at and away from the focal point, allowing shot by shot subtraction of the single photon signal to keep only the non-linear signal.

Acknowledgements: We are grateful to the PF and SOLEIL staff for the stable operation of the synchrotron in top-up mode that allows long time accumulation of the data. NB was funded by DoE BES Division of Chemical Sciences, Geosciences, and Biosciences. These works have been performed with the approval of PF and SOLEIL advisory committee from 2009 to 2014.

\section{$\underline{\text { References }}$}

[1] L. S. Cederbaum, F. Tarantelli, A. Sgamellotti and J Schirmer., J. Chem. Phys., vol. 85, p. 6513, 1986.

[2] L. S. Cederbaum, F. Tarantelli, A. Sgamellotti and J. Schirmer, J. Chem. Phys., vol. 86, p. 2168, 1987.

[3] K. Siegbahn, ESCA Applied to Free Molecules, Amsterdam: North-Holland, 1971.

[4] P. Lablanquie et al, Phys.Rev.Lett., vol. 106, p. 069003, 2011.

[5] J. Eland et al, Phys.Rev.Lett., vol. 105, p. 213005, 2010.

[6] J. H. D. Eland, O. Vieuxmaire, T. Kinugawa, P. Lablanquie, R. I. Hall and F. Penent, Phys. Rev. Lett., vol. 90, p. 053003, 2003.

[7] I. H. Suzuki et al., J. Phys. B: At. Mol. Opt. Phys., vol. 44, p. 075003, 2011.

[8] P. Lablanquie et al, Phys.Rev.Lett., vol. 107, p. 193004, 2011.

[9] M. Nakano et al, Phys.Rev.Lett., vol. 110, p. 163001, 2013.

[10] M. Nakano et al, Phys.Rev.Lett., vol. 111, p. 123001, 2013.

[11] P. Agostini et al, Phys.Rev.Lett., vol. 42, p. 1127, 1979.

[12] M. Lewenstein et al, Phys.Rev.A, vol. 49, p. 2117, 1994.

[13] P. Agostini et L. F. DiMauro, Rep.Prog.Phys., vol. 67, p. 813, 2004 and references therein.

[14] P. Emma et al, Nature Photon., vol. 4, p. 641, 2010.

[15] T. Ishikawa et al, Nature Photon., vol. 6, p. 540, 2012.

[16] M. Žitnik et al, Phys.Rev.Lett., vol. 113, p. 193201, 2014.

[17] F. Biraben et al, Phys.Rev.Lett., vol. 32, p. 643, 1974.

[18] M. Geoppert-Mayer, Annalen der Physik, vol. 401, p. 273, 1931.

[19] G. Doumy et al, Phys.Rev.Lett., vol. 106, p. 083002, 2011.

[20] B. Deschaud, O. Peyrusse et F. B. Rosmej, EPL, vol. 108, p. 53001, 2014.

[21] L. Young et al, Nature, vol. 466, p. 56, 2010.

[22] L. Fang et al, Phys.Rev.Lett, vol. 105, p. 083005, 2010.

[23] N. Berrah et al, Proc.Nat.Acad.Sci.USA, vol. 108, p. 16912, 2011.

[24] J. P. Cryan et al, Phys.Rev.Lett., vol. 105, p. 083004, 2010.

[25] M. Larsson et al, J.Phys.B-At.Mol.Opt.Phys, vol. 46, p. 164030, 2013.

[26] P. Salèn et al, Phys.Rev.Lett., vol. 108, p. 153003, 2012.

[27] M. Tashiro et al, J.Chem.Phys., vol. 132, p. 184302, 2010. 
[28] S. Santra, N. V. Kryzhevoi et L. S. Cederbaum, Phys.Rev.Lett., vol. 103, p. 013002, 2009.

[29] G. Charpak, C.R.Hebd.Scéances.Acad.Sci., vol. 237, p. 243, 1953.

[30] J. Briand et al, J. Phys. B: At.Mol.Phys., vol. 9, p. 1055, 1976.

[31] J. Hoszowska et al, Phys.Rev.Lett., vol. 102, p. 073006, 2009.

[32] J. Hoszowska and J.-C. Dousse, J.Elec.Spectr.Rel.Phenom., vol. 188, p. 62, 2013.

[33] L. J. Frasinski, K. Codling et P. A. Hatherly, Science, vol. 246, p. 1029, 1989.

[34] L. J. Fransinski et al, Phys.Rev.Lett., vol. 111, p. 073002, 2013.

[35] V. Zhaunerchyk et al, J. Phys. B: At. Mol. Opt. Phys., vol. 46, p. 164034, 2013.

[36] V. Zhaunerchyk, L. J. Frasinski, J. Eland and R. Feifel, Phys.Rev.A, vol. 89, p. 053418, 2014.

[37] K. Schnorr et al, Phys.Rev.Lett., vol. 111, p. 093402, 2013.

[38] J. Eland, Chem.Phys., vol. 294, p. 171, 2003.

[39] F. Penent et al, Phys.Rev.Lett., vol. 95, p. 083002, 2005.

[40] J. Palaudoux et al, Phys.Rev.A, vol. 82, p. 043419, 2010.

[41] F. Penent et al, Phys.Rev.Lett., vol. 106, p. 103002, 2011.

[42] Y. Hikosaka et al, Phys.Rev.Lett., vol. 102, p. 013002, 2009.

[43] T. Kanayasu et al, Phys.Rev.A, vol. 76, p. 012717, 2007.

[44] Y. Hikosaka et al, Phys.Rev.Lett., vol. 98, p. 183002, 2007.

[45] M. Mucke et al, Chem.Phys.Lett., vol. 558, p. 82, 2013.

[46] L. Hedin et al, J.Chem.Phys., vol. 140, p. 044309, 2014.

[47] S. Carniato et al, J.Chem.Phys., vol. 142, p. 014307, 2015.

[48] S. Carniato et al, J.Chem.Phys., vol. 142, p. 014308, 2015.

[49] M. Sacchi et al, J. Phys.: Conf. Ser., vol. 425, p. 072018, 2011.

[50] S. Matoba et al, Journal of Physics: Conference Series, vol. 388, p. 142018, 2012.

[51] «http://www.lumat.u-psud.fr/IMG/pdf/dtpi/TDC-V4_User_Manual.pdf,» [En ligne].

[52] K. Ito et al, Rev. Sci. Instrum. 80, vol. 80, p. 123101 , 2009.

[53] T. X. Carrol et al, J.Elec.spectr.Rel.Phenom., vol. 125, p. 127, 2002.

[54] M. Piancastelli, Eur. Phys. J. Special Topics, vol. 222, p. 2035, 2013.

[55] F. Penent et al, J.Elec.Spectr.Rel.Phenom., vol. 196, p. 38, 2014.

[56] M. Tashiro et al, J.Chem.Phys., vol. 137, p. 224306., 2012.

[57] T. Thomas, J.Phys.Chem., vol. 116, p. 3856, 2012.

[58] K. Siegbahn, Rev.Mod.Phys., vol. 54, p. 709, 1982.

[59] H. Iwayama et al, J.Chem.Phys., vol. 138, p. 0243306, 2013.

[60] O. Travnikova et al, J.Elec.Spectr.Rel.Phenom., vol. 185, p. 191, 2012.

[61] S. Carniato et al, in preparation, 2015. 